\title{
A Hierarchy of Discrete Integrable Coupling System with Self-Consistent Sources
}

\author{
Yuqing Li, ${ }^{1}$ Huanhe Dong, ${ }^{1}$ and Baoshu Yin ${ }^{2,3}$ \\ ${ }^{1}$ College of Mathematics and Systems Science, Shandong University of Science and Technology, Qingdao 266590, China \\ ${ }^{2}$ Institute of Oceanology, China Academy of Sciences, Qingdao 266071, China \\ ${ }^{3}$ Key Laboratory of Ocean Circulation and Wave, Chinese Academy of Sciences, Qingdao 266071, China
}

Correspondence should be addressed to Baoshu Yin; baoshuyin@126.com

Received 8 May 2014; Revised 4 August 2014; Accepted 18 August 2014; Published 27 August 2014

Academic Editor: Xinkai Chen

Copyright (c) 2014 Yuqing Li et al. This is an open access article distributed under the Creative Commons Attribution License, which permits unrestricted use, distribution, and reproduction in any medium, provided the original work is properly cited.

Integrable coupling system of a lattice soliton equation hierarchy is deduced. The Hamiltonian structure of the integrable coupling is constructed by using the discrete quadratic-form identity. The Liouville integrability of the integrable coupling is demonstrated. Finally, the discrete integrable coupling system with self-consistent sources is deduced.

\section{Introduction}

Many physical problems may be modeled by soliton equation. The Hamiltonian structures of many systems have been obtained by the famous trace identity [1-6]. The study of integrable couplings of integrable systems has become the focus of common concern in recent years. It originates from the investigations on the symmetry problems and associated centerless Virasoro algebras [7]. Many integrable coupling systems have been constructed by using the methods of a direct method [8], perturbations [9], enlarging spectral problems [10, 11], creating new loop algebras [12, 13], and semidirect sums of Lie algebras [14, 15]. The Hamiltonian structures of the integrable couplings of lattice equations can be constructed by means of the discrete quadratic-form identity $[16,17]$.

Since Mel'Nikov proposed a new kind of integrable model which was called soliton equations with self-consistent sources [18] in 1983, many soliton equations with selfconsistent sources [19-23] have been presented in recent years. For applications, these kinds of systems are usually used to describe interactions between different solitary waves. In this paper, we deduce a hierarchy of discrete integrable coupling system with self-consistent sources which are few compared with the continuous ones.

The paper will be organized as follows. We first get a hierarchy of integrable lattice soliton equation with self-consistent sources in Section 2. In Section 3, a hierarchy of discrete integrable coupling system is derived by making use of the discrete zero curvature representation. By means of the discrete quadratic-form identity we establish the Hamiltonian structures of the hierarchy. Further, the resulting Hamiltonian equations are all proved to be integrable in Liouville sense. Finally, we give the integrable coupling systems with self-consistent sources.

\section{A Hierarchy of Integrable Lattice Soliton Equations with Self-Consistent Sources}

We first briefly describe our notations. Assume $f_{n}=f(n)$ is a lattice function; the shift operator $E$ and the inverse of $E$ are defined by

$$
\begin{gathered}
E f_{n}=f(n+1), \quad E^{-1} f_{n}=f(n-1), \quad n \in Z, \\
E^{k} f_{n}=f(n+k), \quad n, k \in Z .
\end{gathered}
$$

A system of discrete equations

$$
\partial_{t_{m}} u_{n}=K_{m}\left(u_{n}\right)
$$


is said to have a discrete Lax pair

$$
\begin{gathered}
E \varphi_{n}=U_{n}\left(u_{n}, \lambda\right) \varphi_{n}, \\
\partial_{t_{m}} \varphi_{n}=V_{n}^{[m]}\left(u_{n}, \lambda\right) \varphi_{n},
\end{gathered}
$$

if it is equivalent to the compatibility condition

$$
\begin{aligned}
\partial_{t_{m}} & U_{n}\left(u_{n}, \lambda\right) \\
& =\left(E V_{n}^{[m]}\left(u_{n}, \lambda\right)\right) U_{n}\left(u_{n}, \lambda\right)-U_{n}\left(u_{n}, \lambda\right) V_{n}^{[m]}\left(u_{n}, \lambda\right) .
\end{aligned}
$$

In [16], a Lie algebra is presented as

$$
G=\operatorname{span}\left\{\omega_{1}, \omega_{2}, \omega_{3}, \omega_{4}, \omega_{5}, \omega_{6}, \omega_{7}, \omega_{8}\right\},
$$

where

$$
\begin{aligned}
& \omega_{1}=\left(\begin{array}{llll}
1 & 0 & 0 & 0 \\
0 & 0 & 0 & 0 \\
0 & 0 & 1 & 0 \\
0 & 0 & 0 & 0
\end{array}\right), \quad \omega_{2}=\left(\begin{array}{llll}
0 & 0 & 0 & 0 \\
0 & 1 & 0 & 0 \\
0 & 0 & 0 & 0 \\
0 & 0 & 0 & 1
\end{array}\right), \\
& \omega_{3}=\left(\begin{array}{cccc}
0 & 1 & 0 & 0 \\
0 & 0 & 0 & 0 \\
0 & 0 & 0 & 1 \\
0 & 0 & 0 & 0
\end{array}\right), \quad \omega_{4}=\left(\begin{array}{llll}
0 & 0 & 0 & 0 \\
1 & 0 & 0 & 0 \\
0 & 0 & 0 & 0 \\
0 & 0 & 1 & 0
\end{array}\right) \text {, } \\
& \omega_{5}=\left(\begin{array}{cccc}
0 & 0 & 0 & 0 \\
0 & 0 & 0 & 0 \\
1 & 0 & 0 & 0 \\
0 & 0 & 0 & 0
\end{array}\right), \quad \omega_{6}=\left(\begin{array}{llll}
0 & 0 & 0 & 0 \\
0 & 0 & 0 & 0 \\
0 & 0 & 0 & 0 \\
0 & 1 & 0 & 0
\end{array}\right) \text {, } \\
& \omega_{7}=\left(\begin{array}{cccc}
0 & 0 & 0 & 0 \\
0 & 0 & 0 & 0 \\
0 & 0 & 0 & 0 \\
1 & 0 & 0 & 0
\end{array}\right), \quad \omega_{8}=\left(\begin{array}{llll}
0 & 0 & 0 & 0 \\
0 & 0 & 0 & 0 \\
0 & 1 & 0 & 0 \\
0 & 0 & 0 & 0
\end{array}\right) \text {. }
\end{aligned}
$$

Set $G_{1}=\operatorname{span}\left\{\omega_{1}, \omega_{2}, \omega_{3}, \omega_{4}\right\}$ and $G_{2}=\operatorname{span}\left\{\omega_{5}, \omega_{6}, \omega_{7}, \omega_{8}\right\}$; it is easy to see that $G, G_{1}$, and $G_{2}$ construct three Lie algebra, and

$$
G=G_{1} \oplus G_{2}, \quad\left[G_{1}, G_{2}\right] \equiv G_{1} G_{2}-G_{2} G_{1} \subseteq G_{2} .
$$

So $G_{2}$ is an Abelian ideal of the Lie algebra $G$. The corresponding loop algebra $\widetilde{G}$ is defined by

$$
\widetilde{G}=\operatorname{span}\left\{\omega_{i}(m), i=1,2, \ldots, 8\right\}, \quad \omega_{i}(m)=\omega_{i} \lambda^{m} .
$$

In [15], a new discrete matrix spectral problem has been proposed:

$$
E \phi_{n}=\widetilde{U}_{n}\left(r_{n}, \lambda\right) \phi_{n}, \quad \widetilde{U}_{n}\left(r_{n}, \lambda\right)=r_{n} \omega_{2}(1)+\omega_{3}(0),
$$

by solving the stationary discrete zero curvature equation

$$
\left(E \Gamma_{n}\right) U_{n}-U_{n} \Gamma_{n}=0,
$$

where

$$
\begin{gathered}
\Gamma_{n}=a_{n} \omega_{1}(0)-a_{n} \omega_{2}(0)+b_{n} \omega_{3}(0)+c_{n} \omega_{4}(1), \\
a_{n}=\sum_{m=0}^{\infty} a_{n}^{(m)} \lambda^{-m}, \quad b_{n}=\sum_{m=0}^{\infty} b_{n}^{(m)} \lambda^{-m}, \\
c_{n}=\sum_{m=0}^{\infty} c_{n}^{(m)} \lambda^{-m},
\end{gathered}
$$

and introducing the auxiliary spectral problems associated with the spectral problem (9)

$$
\begin{gathered}
\partial_{t_{m}} \phi_{n}=\widetilde{V}_{n}^{[m]} \phi_{n}, \quad m \geq 0 \\
\widetilde{V}_{n}^{[m]}=\sum_{i=0}^{m}\left[a_{n}^{(i)} \omega_{1}(m-i)-a_{n}^{(i)} \omega_{2}(m-i)\right. \\
\left.+b_{n}^{(i)} \omega_{3}(m-i)+c_{n}^{(i)} \omega_{4}(m-i+1)\right] \\
-a_{n}^{(m)} \omega_{1}(0)+a_{n}^{(m)} \omega_{2}(0),
\end{gathered}
$$

a hierarchy of integrable lattice soliton equations with a potential $r_{n}$ has been presented:

$$
\partial_{t_{m}} r_{n}=r_{n}\left(a_{n+1}^{(m)}-a_{n}^{(m)}\right), \quad m \geq 0
$$

where

$$
\begin{gathered}
a_{n}^{(0)}=-\frac{1}{2}, \quad a_{n}^{(1)}=\frac{1}{r_{n} r_{n-1}} \\
a_{n}^{(2)}=-\frac{1}{r_{n} r_{n-1}}\left(\frac{1}{r_{n} r_{n-1}}+\frac{1}{r_{n-2} r_{n-1}}+\frac{1}{r_{n+1} r_{n}}\right), \ldots
\end{gathered}
$$

Equation (13) possesses the following Hamiltonian forms [15]:

$$
\partial_{t_{m}} r_{n}=\widetilde{J} \frac{\delta \widetilde{F}_{n}^{(m)}}{\delta r_{n}}=\widetilde{M} \frac{\delta \widetilde{F}_{n}^{(m-1)}}{\delta r_{n}}, \quad m \geq 1,
$$

where

$$
\begin{gathered}
\widetilde{J}=r_{n}(1-E)(1+E)^{-1} r_{n}, \quad \widetilde{M}=E-E^{-1}, \\
\widetilde{F}_{n}^{(m)}=\sum_{n \in z} F_{n}^{(m)}, \quad F_{n}^{(m)}=-\frac{a_{n}^{(m)}}{m}, \quad m \geq 1 .
\end{gathered}
$$

Next, we will construct a hierarchy of integrable lattice soliton equations (13) with self-consistent sources. For $n$ distinct real $\lambda_{j}$, consider the auxiliary linear problem

$$
\begin{gathered}
E\left(\begin{array}{l}
\phi_{1 j} \\
\phi_{2 j} \\
\phi_{3 j} \\
\phi_{4 j}
\end{array}\right)=\widetilde{U}_{n}\left(r_{n}, \lambda_{j}\right)\left(\begin{array}{l}
\phi_{1 j} \\
\phi_{2 j} \\
\phi_{3 j} \\
\phi_{4 j}
\end{array}\right), \\
\left(\begin{array}{c}
\phi_{1 j} \\
\phi_{2 j} \\
\phi_{3 j} \\
\phi_{4 j}
\end{array}\right)_{t_{m}}=\widetilde{V}_{n}\left(r_{n}, \lambda_{j}\right)\left(\begin{array}{c}
\phi_{1 j} \\
\phi_{2 j} \\
\phi_{3 j} \\
\phi_{4 j}
\end{array}\right) .
\end{gathered}
$$

Based on the results in [24], we show the following equation:

$$
\frac{\delta \widetilde{F}_{n}^{(m)}}{\delta r_{n}}+\sum_{j=1}^{N} \frac{\delta \lambda_{j}}{\delta r_{n}}=0,
$$


where

$$
\begin{gathered}
\frac{\delta \lambda_{j}}{\delta r_{n}}=\frac{1}{2} \operatorname{Tr}\left(\psi_{j} \frac{\partial \widetilde{U}\left(r_{n}, \lambda_{j}\right)}{\partial r_{n}}\right), \\
\psi_{j}=\left(\begin{array}{cccc}
\phi_{1 j} \phi_{2 j} & -\phi_{1 j}^{2} & \phi_{3 j} \phi_{4 j} & -\phi_{3 j}^{2} \\
\phi_{2 j}^{2} & -\phi_{1 j} \phi_{2 j} & \phi_{4 j}^{2} & -\phi_{3 j} \phi_{4 j} \\
0 & 0 & \phi_{1 j} \phi_{2 j} & -\phi_{1 j}^{2} \\
0 & 0 & \phi_{2 j}^{2} & -\phi_{1 j} \phi_{2 j}
\end{array}\right), \\
\\
\end{gathered}
$$

According to the approach proposed in [24-26], through a direct computation, we obtain the discrete integrable hierarchy with self-consistent sources as follows:

$$
\begin{aligned}
\partial_{t_{m}} r_{n} & =\widetilde{J}\left(\frac{\delta \widetilde{F}_{n}^{(m)}}{\delta r_{n}}+\sum_{j=1}^{N} \frac{\delta \lambda_{j}}{\delta r_{n}}\right) \\
& =\widetilde{J}\left(\frac{\delta \widetilde{F}_{n}^{(m)}}{\delta r_{n}}-\sum_{j=1}^{N} \lambda_{j} \phi_{1 j} \phi_{2 j}\right), \quad m \geq 1 .
\end{aligned}
$$

Taking $m=1$ in the above system, under $t_{1} \rightarrow t$, we can obtain the following equation with self-consistent sources:

$$
\partial_{t} r_{n}=\frac{1}{r_{n+1}}-\frac{1}{r_{n-1}}-r_{n}(1-E)(1+E)^{-1} r_{n} \sum_{j=1}^{N} \lambda_{j} \phi_{1 j} \phi_{2 j} .
$$

\section{A Hierarchy of Discrete Integrable Coupling System with Self-Consistent Sources}

First, we will give out the integrable couplings of the hierarchy (13). Consider the discrete isospectral problem

$$
\begin{aligned}
E \phi_{n} & =U_{n}\left(u_{n}, \lambda\right) \phi_{n}, \\
U_{n}\left(u_{n}, \lambda\right) & =r_{n} \omega_{2}(1)+\omega_{3}(0)+\omega_{4}(1)+s_{n} \omega_{6}(1),
\end{aligned}
$$

in which $u_{n}=\left(r_{n}, s_{n}\right)^{T}$ is the potential, $r_{n}=r(n, t)$ and $s_{n}=$ $s(n, t)$ are real functions defined over $Z \times R, \lambda$ is a spectral parameter, $\lambda_{t}=0$, and $\phi_{n}=\left(\phi_{1}(n), \phi_{2}(n), \phi_{3}(n), \phi_{4}(n)\right)^{T}$ is the eigenfunction vector.

We solve the stationary discrete zero curvature equation

$$
\left(E \Gamma_{n}\right) U_{n}-U_{n} \Gamma_{n}=0,
$$

where

$$
\begin{aligned}
\Gamma_{n}= & a_{n} \omega_{1}(0)-a_{n} \omega_{2}(0)+b_{n} \omega_{3}(0)+c_{n} \omega_{4}(1) \\
& +e_{n} \omega_{5}(0)-e_{n} \omega_{6}(0)+g_{n} \omega_{7}(1)+f_{n} \omega_{8}(0) .
\end{aligned}
$$

Equation (23) gives

$$
\begin{gathered}
b_{n+1}=c_{n}, \\
a_{n}+\lambda r_{n} b_{n+1}+a_{n+1}=0, \\
a_{n}+\lambda r_{n} c_{n}+a_{n+1}=0, \\
c_{n+1}-b_{n}+r_{n}\left(a_{n}-a_{n+1}\right)=0, \\
f_{n+1}=g_{n}, \\
e_{n+1}+e_{n}+\lambda r_{n} f_{n+1}+\lambda s_{n} b_{n+1}=0, \\
e_{n+1}+e_{n}+\lambda r_{n} g_{n}+\lambda s_{n} c_{n}=0, \\
-f_{n}+g_{n+1}+r_{n}\left(e_{n}-e_{n+1}\right)+s_{n}\left(a_{n}-a_{n+1}\right)=0 .
\end{gathered}
$$

Substituting the expansions

$$
\begin{array}{rlrl}
a_{n} & =\sum_{m=0}^{\infty} a_{n}^{(m)} \lambda^{-m}, & b_{n} & =\sum_{m=0}^{\infty} b_{n}^{(m)} \lambda^{-m}, \\
c_{n}=\sum_{m=0}^{\infty} c_{n}^{(m)} \lambda^{-m}, & e_{n}=\sum_{m=0}^{\infty} e_{n}^{(m)} \lambda^{-m}, \\
f_{n}=\sum_{m=0}^{\infty} f_{n}^{(m)} \lambda^{-m}, & g_{n}=\sum_{m=0}^{\infty} g_{n}^{(m)} \lambda^{-m}
\end{array}
$$

into (25), we can get the recursion relation

$$
\begin{gathered}
a_{n+1}^{(m)}+a_{n}^{(m)}=-r_{n} b_{n+1}^{(m+1)}, \\
a_{n+1}^{(m)}+a_{n}^{(m)}=-r_{n} c_{n}^{(m+1)}, \\
r_{n}\left(a_{n}^{m}-a_{n+1}^{(m)}\right)+c_{n+1}^{(m)}-b_{n}^{m}=0, \\
e_{n+1}^{(m)}+e_{n}^{(m)}=-s_{n} b_{n+1}^{(m+1)}-r_{n} f_{n+1}^{(m+1)}, \\
e_{n+1}^{(m)}+e_{n}^{(m)}=-s_{n} c_{n}^{(m+1)}-r_{n} g_{n}^{(m+1)}, \\
s_{n}\left(a_{n}^{(m)}-a_{n+1}^{(m)}\right)+r_{n}\left(e_{n}^{(m)}-e_{n+1}^{(m)}\right)-f_{n}^{(m)}+g_{n+1}^{(m)}=0 .
\end{gathered}
$$

The initial values are taken as

$$
\begin{array}{lll}
a_{n}^{(0)}=-\frac{1}{2}, & b_{n}^{(0)}=0, & c_{n}^{(0)}=0, \\
e_{n}^{(0)}=-\frac{1}{2}, & f_{n}^{(0)}=0, & g_{n}^{(0)}=0 .
\end{array}
$$

Note that the definition of the inverse operator of $D=(E-$ $1)$ does not yield any arbitrary constant in computing $a_{n}^{(m)}$ and $e_{n}^{(m)}, m \geq 1$. Thus, the recursion relation (27) uniquely determines

$$
a_{n}^{(m)}, b_{n}^{(m)}, c_{n}^{(m)}, e_{n}^{(m)}, f_{n}^{(m)}, g_{n}^{(m)}, \quad m \geq 1,
$$


and the first few quantities are given by

$$
\begin{aligned}
& a_{n}^{(1)}=\frac{1}{r_{n} r_{n-1}}, \quad b_{n}^{(1)}=\frac{1}{r_{n-1}}, \quad c_{n}^{(1)}=\frac{1}{r_{n}}, \\
& e_{n}^{(1)}=\frac{1}{r_{n-1} r_{n}}-\frac{s_{n-1}}{r_{n-1}^{2} r_{n}}-\frac{s_{n}}{r_{n}^{2} r_{n-1}}, \quad f_{n}^{(1)}=\frac{1}{r_{n-1}}-\frac{s_{n-1}}{r_{n-1}^{2}}, \\
& g_{n}^{(1)}=\frac{1}{r_{n}}-\frac{s_{n}}{r_{n}^{2}} \\
& a_{n}^{(2)}=-\frac{1}{r_{n} r_{n-1}}\left(\frac{1}{r_{n} r_{n-1}}+\frac{1}{r_{n-2} r_{n-1}}+\frac{1}{r_{n+1} r_{n}}\right) \text {, } \\
& b_{n}^{(2)}=-\frac{1}{r_{n-1}^{2}}\left(\frac{1}{r_{n-2}}+\frac{1}{r_{n}}\right), \quad c_{n}^{(2)}=-\frac{1}{r_{n}^{2}}\left(\frac{1}{r_{n-1}}+\frac{1}{r_{n+1}}\right) \text {, } \\
& e_{n}^{(2)}=-\frac{1}{r_{n} r_{n-1}}\left(\frac{1}{r_{n} r_{n-1}}+\frac{1}{r_{n} r_{n+1}}+\frac{1}{r_{n-2} r_{n-1}}\right) \\
& +\frac{2 s_{n}}{r_{n-1} r_{n}^{3}}\left(\frac{1}{r_{n+1}}+\frac{1}{r_{n-1}}\right)+\frac{2 s_{n-1}}{r_{n} r_{n-1}^{3}}\left(\frac{1}{r_{n-2}}+\frac{1}{r_{n}}\right) \\
& +\frac{1}{r_{n}^{2} r_{n+1}^{2}}\left(\frac{s_{n-1}}{r_{n+2}}+\frac{s_{n+1}}{r_{n-1}}\right)+\frac{1}{r_{n-1}^{2} r_{n} r_{n-2}}\left(\frac{s_{n-2}}{r_{n-2}}+\frac{s_{n}}{r_{n}}\right) \text {, } \\
& f_{n}^{(2)}=\frac{2 s_{n-1}}{r_{n-1}^{2}}\left(\frac{1}{r_{n}}+\frac{1}{r_{n-2}}\right)+\frac{1}{r_{n-1}^{2} r_{n-2}}\left(\frac{s_{n-2}}{r_{n-2}}-1\right) \\
& +\frac{1}{r_{n-1}^{2} r_{n}}\left(\frac{s_{n}}{r_{n}}-1\right) \\
& g_{n}^{(2)}=\frac{2 s_{n}}{r_{n}^{2}}\left(\frac{1}{r_{n+1}}+\frac{1}{r_{n-1}}\right)+\frac{1}{r_{n}^{2} r_{n-1}}\left(\frac{s_{n-1}}{r_{n-1}}-1\right) \\
& +\frac{1}{r_{n}^{2} r_{n+1}}\left(\frac{s_{n+1}}{r_{n+1}}-1\right) \text {. }
\end{aligned}
$$

Set

$$
\begin{aligned}
V_{n}^{(m)}=\sum_{i=0}^{m}[ & a_{n}^{(i)} \omega_{1}(m-i)-a_{n}^{(i)} \omega_{2}(m-i)+b_{n}^{(i)} \omega_{3}(m-i) \\
& +c_{n}^{(i)} \omega_{4}(m-i+1)+e_{n}^{(i)} \omega_{5}(m-i)-e_{n}^{(i)} \omega_{6} \\
& \left.\times(m-i)+g_{n}^{(i)} \omega_{7}(m-i+1)+f_{n}^{(i)} \omega_{8}(m-i)\right],
\end{aligned}
$$

so

$$
\begin{aligned}
E\left(V_{n}^{(m)}\right) U_{n}-U_{n} V_{n}^{(m)} & \\
= & -r_{n} c_{n}^{(m+1)} \omega_{3}(0)+r_{n} c_{n}^{(m+1)} \omega_{4}(1)-\left(e_{n}^{(m)}+e_{n+1}^{(m)}\right) \omega_{7}(1) \\
& +\left(e_{n}^{(m)}+e_{n+1}^{(m)}\right) \omega_{8}(0)
\end{aligned}
$$

Take $\eta_{n}^{(m)}=-a_{n}^{(m)} \omega_{1}(0)+a_{n}^{(m)} \omega_{2}(0)-e_{n}^{(m)} \omega_{5}(0)+e_{n}^{(m)} \omega_{6}(0)$, $m \geq 0$, and let

$$
V_{n}^{[m]}=V_{n}^{(m)}+\eta_{n}^{(m)}
$$

We introduce the auxiliary spectral problems associated with the spectral problem (22):

$$
\partial_{t_{m}} \phi_{n}=V_{n}^{[m]} \phi_{n}, \quad m \geq 0
$$

The compatibility conditions of (22) and (34) are

$$
\partial_{t_{m}} U_{n}=\left(E V_{n}^{[m]}\right) U_{n}-U_{n} V_{n}^{[m]}, \quad m \geq 0
$$

which give rise to the following hierarchy of integrable lattice equations:

$$
\begin{gathered}
\partial_{t_{m}} r_{n}=r_{n}\left(a_{n+1}^{(m)}-a_{n}^{(m)}\right), \quad m \geq 0, \\
\partial_{t_{m}} s_{n}=s_{n}\left(a_{n+1}^{(m)}-a_{n}^{(m)}\right)-r_{n}\left(e_{n}^{(m)}-e_{n+1}^{(m)}\right), \quad m \geq 0 .
\end{gathered}
$$

So (35) is the discrete zero curvature representation of (36); the discrete spectral problems (22) and (34) constitute the Lax pairs of (36), and (36) are a hierarchy of Lax integrable nonlinear lattice equations. It is easy to verify that the first nonlinear lattice equation in (36), when $m=1$, under $t_{1} \rightarrow t$, is

$$
\begin{gathered}
\partial_{t} r_{n}=\left(E-E^{-1}\right) \frac{1}{r_{n}}, \\
\partial_{t} s_{n}=\left(E^{-1}-E\right) \frac{s_{n}}{r_{n}^{2}}+\left(E-E^{-1}\right) \frac{1}{r_{n}} .
\end{gathered}
$$

In (36) the first lattice equations

$$
\partial_{t_{m}} r_{n}=r_{n}\left(a_{n+1}^{(m)}-a_{n}^{(m)}\right), \quad m \geq 0
$$

constitute a hierarchy of integrable lattice soliton equations with a potential $r_{n}$; in the view of integrable coupling theory $[7,13,17],(36)$ are integrable coupling systems of (13) or (15).

In what follows, we would like to establish the Hamiltonian structures for the integrable coupling systems (36).

Set $a=\sum_{i=1}^{8} a_{i} \omega_{i}, b=\sum_{i=1}^{8} b_{i} \omega_{i}$, and $c=\sum_{i=1}^{8} c_{i} \omega_{i} \in G$. We define a map

$$
\sigma: G \longrightarrow R^{8}, \quad a \longmapsto\left(a_{1}, a_{2}, \ldots, a_{8}\right)^{T}, \quad a \in G .
$$

Following [16], we introduce the matrix

$$
F=\left(\begin{array}{llllllll}
1 & 0 & 0 & 0 & 1 & 0 & 0 & 0 \\
0 & 1 & 0 & 0 & 0 & 1 & 0 & 0 \\
0 & 0 & 0 & 1 & 0 & 0 & 1 & 0 \\
0 & 0 & 1 & 0 & 0 & 0 & 0 & 1 \\
1 & 0 & 0 & 0 & 0 & 0 & 0 & 0 \\
0 & 1 & 0 & 0 & 0 & 0 & 0 & 0 \\
0 & 0 & 1 & 0 & 0 & 0 & 0 & 0 \\
0 & 0 & 0 & 1 & 0 & 0 & 0 & 0
\end{array}\right)
$$

It is easy to verify that $F$ meets $F^{T}=F$. Under the definition of the quadratic-form function

$$
\{a, b\}=a^{T} F b
$$


we have $\{a b, c\}=\{a, b c\}$ and $a, b, c \in G$. Set $R_{n}=\Gamma_{n} U_{n}^{-1}$; through a direct calculation, we get

$$
\begin{gathered}
\left\{R_{n}, \frac{\partial U_{n}}{\partial \lambda}\right\}=\frac{e_{n}+a_{n}}{\lambda}+r_{n}\left(c_{n}+g_{n}\right)+s_{n} c_{n}, \\
\left\{R_{n}, \frac{\partial U_{n}}{\partial r_{n}}\right\}=\lambda\left(c_{n}+g_{n}\right), \quad\left\{R_{n}, \frac{\partial U_{n}}{\partial s_{n}}\right\}=\lambda c_{n} .
\end{gathered}
$$

By the discrete quadratic-form identity [16]

$$
\begin{aligned}
& \frac{\delta}{\delta r_{n}} \sum_{n \in Z}\left\{R_{n}, \frac{\partial U_{n}}{\partial \lambda}\right\}=\left(\lambda^{-\gamma}\left(\frac{\partial}{\partial \lambda}\right) \lambda^{\gamma}\right)\left\{R_{n}, \frac{\partial U_{n}}{\partial r_{n}}\right\}, \\
& \frac{\delta}{\delta s_{n}} \sum_{n \in Z}\left\{R_{n}, \frac{\partial U_{n}}{\partial \lambda}\right\}=\left(\lambda^{-\gamma}\left(\frac{\partial}{\partial \lambda}\right) \lambda^{\gamma}\right)\left\{R_{n}, \frac{\partial U_{n}}{\partial s_{n}}\right\},
\end{aligned}
$$

with $\gamma$ being a constant to be determined, we have

$$
\begin{aligned}
& \frac{\delta}{\delta r_{n}} \sum_{n \in Z}\left[\frac{e_{n}+a_{n}}{\lambda}+r_{n}\left(c_{n}+g_{n}\right)+s_{n} c_{n}\right] \\
& =\lambda^{-\lambda}\left(\frac{\partial}{\partial \lambda}\right) \lambda^{\gamma}\left[\lambda\left(c_{n}+g_{n}\right)\right], \\
& \frac{\delta}{\delta s_{n}} \sum_{n \in Z}\left[\frac{e_{n}+a_{n}}{\lambda}+r_{n}\left(c_{n}+g_{n}\right)+s_{n} c_{n}\right] \\
& =\lambda^{-\lambda}\left(\frac{\partial}{\partial \lambda}\right) \lambda^{\gamma}\left(\lambda c_{n}\right) .
\end{aligned}
$$

By the substitution of

$$
\begin{array}{ll}
a_{n}=\sum_{m=0}^{\infty} a_{n}^{(m)} \lambda^{-m}, & b_{n}=\sum_{m=0}^{\infty} b_{n}^{(m)} \lambda^{-m}, \\
c_{n}=\sum_{m=0}^{\infty} c_{n}^{(m)} \lambda^{-m}, & e_{n}=\sum_{m=0}^{\infty} e_{n}^{(m)} \lambda^{-m} \\
f_{n}=\sum_{m=0}^{\infty} f_{n}^{(m)} \lambda^{-m}, & g_{n}=\sum_{m=0}^{\infty} g_{n}^{(m)} \lambda^{-m}
\end{array}
$$

into (44) and comparing the coefficients of $\lambda^{-m-1}$ in (44), we get

$$
\begin{aligned}
& \left(\begin{array}{c}
\frac{\delta}{\delta r_{n}} \\
\frac{\delta}{\delta s_{n}}
\end{array}\right) \sum_{n \in Z}\left[e_{n}^{(m)}+a_{n}^{(m)}+r_{n}\left(c_{n}^{(m+1)}+g_{n}^{(m+1)}\right)+s_{n} c_{n}^{(m+1)}\right] \\
& =(-m+\gamma)\left(\begin{array}{c}
c_{n}^{(m+1)}+g_{n}^{(m+1)} \\
c_{n}^{(m+1)}
\end{array}\right) .
\end{aligned}
$$

Now we can rewrite those lattice equations in (36) as

$$
\left(\begin{array}{l}
r_{n} \\
s_{n}
\end{array}\right)_{t_{m}}=J\left(\begin{array}{c}
\frac{\delta \widetilde{H}_{n}^{(m)}}{\delta r_{n}} \\
\frac{\delta \widetilde{H}_{n}^{(m)}}{\delta s_{n}}
\end{array}\right),
$$

where $J$ is a local difference operator defined by

$$
J=\left(\begin{array}{ll}
J_{11} & J_{12} \\
J_{21} & J_{22}
\end{array}\right)
$$

where

$$
\begin{gathered}
J_{11}=0, \\
J_{12}=J_{21}=r_{n}(1+E)^{-1}(1-E) r_{n}, \\
J_{22}=s_{n}(1+E)^{-1}(1-E) r_{n}+r_{n}(1+E)^{-1}(1-E) s_{n} \\
+r_{n}(1+E)^{-1}(E-1) r_{n} .
\end{gathered}
$$

Obviously, the operator $J$ is a skew-symmetric operator; that is, $J^{*}=-J$. Moreover, we can prove that the operator $J$ satisfies the Jacobi identity

$$
\left\langle J^{\prime}\left(u_{n}\right)\left[J f_{n}\right] g_{n}, h_{n}\right\rangle+\operatorname{Cycle}\left(f_{n}, g_{n}, h_{n}\right)=0 .
$$

So we have the following facts. 
Proposition 1. $J$ is a discrete Hamiltonian operator.

Set

$$
\frac{\delta \widetilde{H}_{n}^{(m)}}{\delta u_{n}}=\zeta_{n} \frac{\delta \widetilde{H}_{n}^{(m-1)}}{\delta u_{n}}
$$

From the recursion relation (27) we can get the recursion operator $\zeta_{n}$ in (53).

Therefore, we have

$$
\left(\begin{array}{l}
r_{n} \\
s_{n}
\end{array}\right)_{t_{m}}=J \frac{\delta \widetilde{H}_{n}^{(m)}}{\delta u_{n}}=J \zeta_{n} \frac{\delta \widetilde{H}_{n}^{(m-1)}}{\delta u_{n}}=J \zeta_{n}^{m} \frac{\delta \widetilde{H}_{n}^{(0)}}{\delta u_{n}}, \quad m \geq 0
$$

So (49) are a family of Hamiltonian systems. The hierarchy of lattice equations (36) possesses Hamiltonian structures (54). Furthermore, a direct calculation shows that

$$
M=J \zeta_{n}=\left(\begin{array}{cc}
0 & E-E^{-1} \\
E-E^{-1} & E^{-1}-E
\end{array}\right)
$$

It is easy to verify that the operator $M$ is a skew-symmetric operator; that is, $M^{*}=-M$. So we have the following.

Proposition 2. $\left\{\widetilde{H}_{n}^{(m)}\right\}_{m \geq 1}$ defined by (48) forms an infinite set of conserved functionals of the hierarchy (36), and $\widetilde{H}_{n}^{(m)}, m \geq 1$, are involution in pairs with respect to the Poisson bracket.

Proof. We can find that $M^{*}=-M$. Namely, $\left(J \zeta_{n}\right)^{*}=-J \zeta_{n}$, and then $\zeta_{n}^{*} J=J \zeta_{n}$. Hence

$$
\begin{aligned}
\left\{\widetilde{H}_{n}^{(m)}, \widetilde{H}_{n}^{(l)}\right\}_{J} & =\left\langle\frac{\delta \widetilde{H}_{n}^{(m)}}{\delta u_{n}}, J \frac{\delta \widetilde{H}_{n}^{(l)}}{\delta u_{n}}\right\rangle \\
& =\left\langle\zeta_{n}^{m-1} \frac{\delta \widetilde{H}_{n}^{(1)}}{\delta u_{n}}, J \zeta_{n}^{l-1} \frac{\delta \widetilde{H}_{n}^{(1)}}{\delta u_{n}}\right\rangle \\
& =\left\langle\zeta_{n}^{m-1} \frac{\delta \widetilde{H}_{n}^{(1)}}{\delta u_{n}}, \zeta_{n}^{*} J \zeta_{n}^{l-2} \frac{\delta \widetilde{H}_{n}^{(1)}}{\delta u_{n}}\right\rangle \\
& =\left\langle\zeta_{n}^{m} \frac{\delta \widetilde{H}_{n}^{(1)}}{\delta u_{n}}, J \zeta_{n}^{l-2} \frac{\delta \widetilde{H}_{n}^{(1)}}{\delta u_{n}}\right\rangle \\
& =\left\{\widetilde{H}_{n}^{(m+1)}, \widetilde{H}_{n}^{(l-1)}\right\}_{J}=\cdots=\left\{\widetilde{H}_{n}^{(m+l-1)}, \widetilde{H}_{n}^{(1)}\right\}_{J} .
\end{aligned}
$$

Similarly, we get

$$
\left\{\widetilde{H}_{n}^{(l)}, \widetilde{H}_{n}^{(m)}\right\}_{J}=\left\{\widetilde{H}_{n}^{(m+l-1)}, \widetilde{H}_{n}^{(1)}\right\}_{J}
$$

This implies that

$$
\left\{\widetilde{H}_{n}^{(l)}, \widetilde{H}_{n}^{(m)}\right\}_{J}=-\left\{\widetilde{H}_{n}^{(m)}, \widetilde{H}_{n}^{(l)}\right\}_{J}
$$

Thus

$$
\begin{aligned}
\left\{\widetilde{H}_{n}^{(m)}, \widetilde{H}_{n}^{(l)}\right\}_{J}=0, \quad m, l \geq 1, \\
\left(\widetilde{H}_{n}^{(m)}\right)_{t_{l}}=\left\langle\frac{\delta \widetilde{H}_{n}^{(m)}}{\delta u_{n}}, u_{n t_{l}}\right\rangle=\left\langle\frac{\delta \widetilde{H}_{n}^{(m)}}{\delta u_{n}}, J \frac{\delta \widetilde{H}_{n}^{(l)}}{\delta u_{n}}\right\rangle \\
=\left\{\widetilde{H}_{n}^{(m)}, \widetilde{H}_{n}^{(l)}\right\}_{J}=0, \quad m, l \geq 1 .
\end{aligned}
$$

In summary, we obtain the following theorem.

Theorem 3. The lattice equations in (36) or the discrete Hamiltonian equations in (49) are all discrete Liouville integrable Hamiltonian systems.

Now we search for the integrable coupling systems with self-consistent sources. For $n$ distinct real $\lambda_{j}$, consider the auxiliary linear problem

$$
\begin{gathered}
E\left(\begin{array}{l}
\phi_{1 j} \\
\phi_{2 j} \\
\phi_{3 j} \\
\phi_{4 j}
\end{array}\right)=U_{n}\left(u_{n}, \lambda_{j}\right)\left(\begin{array}{l}
\phi_{1 j} \\
\phi_{2 j} \\
\phi_{3 j} \\
\phi_{4 j}
\end{array}\right), \\
\left(\begin{array}{c}
\phi_{1 j} \\
\phi_{2 j} \\
\phi_{3 j} \\
\phi_{4 j}
\end{array}\right)_{t_{m}}=V_{n}^{[m]}\left(u_{n}, \lambda_{j}\right)\left(\begin{array}{c}
\phi_{1 j} \\
\phi_{2 j} \\
\phi_{3 j} \\
\phi_{4 j}
\end{array}\right) .
\end{gathered}
$$

Based on the results in [24], we show the following equation:

$$
\frac{\delta H_{n}^{(m)}}{\delta u_{n}}+\sum_{j=1}^{N} \frac{\delta \lambda_{j}}{\delta u_{n}}=0
$$

where

$$
\begin{aligned}
& \frac{\delta \lambda_{j}}{\delta u_{n}}=\frac{1}{2} \operatorname{Tr}\left(\psi_{j} \frac{\partial U\left(u_{n}, \lambda_{j}\right)}{\partial u_{n}}\right), \\
& \psi_{j}=\left(\begin{array}{cccc}
\phi_{1 j} \phi_{2 j} & -\phi_{1 j}^{2} & \phi_{3 j} \phi_{4 j} & -\phi_{3 j}^{2} \\
\phi_{2 j}^{2} & -\phi_{1 j} \phi_{2 j} & \phi_{4 j}^{2} & -\phi_{3 j} \phi_{4 j} \\
0 & 0 & \phi_{1 j} \phi_{2 j} & -\phi_{1 j}^{2} \\
0 & 0 & \phi_{2 j}^{2} & -\phi_{1 j} \phi_{2 j}
\end{array}\right) \text {, } \\
& j=1,2, \ldots, N \text {. }
\end{aligned}
$$

According to the approach proposed in [24-26], through a direct computation, we get the discrete integrable hierarchy with self-consistent sources as follows: 


$$
\begin{aligned}
\left(\begin{array}{l}
r_{n} \\
s_{n}
\end{array}\right)_{t_{m}}=J\left(\begin{array}{c}
\frac{\delta \widetilde{H}_{n}^{(m)}}{\delta r_{n}}+\sum_{j=1}^{N} \frac{\delta \lambda_{j}}{\delta r_{n}} \\
\frac{\delta \widetilde{H}_{n}^{(m)}}{\delta s_{n}}+\sum_{j=1}^{N} \frac{\delta \lambda_{j}}{\delta s_{n}}
\end{array}\right) \\
=J\left(\begin{array}{c}
\frac{\delta \widetilde{H}_{n}^{(m)}}{\delta r_{n}}-\sum_{j=1}^{N} \lambda_{j} \phi_{1 j} \phi_{2 j} \\
\frac{\delta \widetilde{H}_{n}^{(m)}}{\delta s_{n}}-\sum_{j=1}^{N} \lambda_{j} \phi_{3 j} \phi_{4 j}
\end{array}\right), \quad m \geq 0 .
\end{aligned}
$$

When $m=1$ in the above system, under $t_{1} \rightarrow t$, we can obtain the following coupling equations with self-consistent sources:

$$
\begin{aligned}
\partial_{t} r_{n}= & \left(E-E^{-1}\right) \frac{1}{r_{n}} \\
& -r_{n}(1-E)(1+E)^{-1} r_{n} \sum_{j=1}^{N} \lambda_{j} \phi_{1 j} \phi_{2 j}, \\
\partial_{t} s_{n}= & \left(E^{-1}-E\right) \frac{s_{n}}{r_{n}^{2}}+\left(E-E^{-1}\right) \frac{1}{r_{n}}-r_{n}(1-E) \\
& \times(1+E)^{-1} r_{n}\left(\sum_{j=1}^{N} \lambda_{j} \phi_{1 j} \phi_{2 j}+\sum_{j=1}^{N} \lambda_{j} \phi_{3 j} \phi_{4 j}\right) \\
& -\left[s_{n}(1+E)^{-1}(1-E) r_{n}+r_{n}(1+E)^{-1}(1-E) s_{n}\right] \\
& \times \sum_{j=1}^{N} \lambda_{j} \phi_{3 j} \phi_{4 j} .
\end{aligned}
$$

\section{Conflict of Interests}

The authors declare that there is no conflict of interests regarding the publication of this paper.

\section{Acknowledgments}

This work was supported by the Global Change and AirSea Interaction (no. GASI-03-01-01-02), National Natural Science Foundation of China (no. 61304074), the Nature Science Foundation of Shandong Province of China (no. ZR2013AQ017), the Science and Technology Plan Project of Qingdao (no. 14-2-4-77-JCH), the Open Fund of the Key Laboratory of Ocean Circulation and Waves, the Chinese Academy of Science (no. KLOCAW1401), the Open Fund of the Key Laboratory of Data Analysis and Application, and the State Oceanic Administration (no. LDAA-2013-04).

\section{References}

[1] M. J. Ablowitz and J. F. Ladik, "Nonlinear differential-difference equations," Journal of Mathematical Physics, vol. 16, pp. 598-603, 1975.
[2] G. Z. Tu, "A trace identity and its applications to the theory of discrete integrable systems," Journal of Physics A: Mathematical and General, vol. 23, no. 17, pp. 3903-3922, 1990.

[3] Y. F. Zhang, "A generalized Boite-Pempinelli-Tu (BPT) hierarchy and its bi-Hamiltonian structure," Physics Letters A, vol. 317, no. 3-4, pp. 280-286, 2003.

[4] X. R. Wang, Y. Fang, and H. H. Dong, "Component-trace identity for Hamiltonian structure of the integrable couplings of the Giachetti-Johnson (GJ) hierarchy and coupling integrable couplings," Communications in Nonlinear Science and Numerical Simulation, vol. 16, no. 7, pp. 2680-2688, 2011.

[5] H.-H. Dong, "A new loop algebra and two new Liouville integrable hierarchy," Modern Physics Letters B, vol. 21, no. 11, pp. 663-673, 2007.

[6] H. H. Dong, "A subalgebra of Lie algebra $A_{2}$ and its associated two types of loop algebras, as well as Hamiltonian structures of integrable hierarchy," Journal of Mathematical Physics, vol. 50, no. 5, Article ID 053519, pp. 2899-2905, 2009.

[7] W. X. Ma and B. Fuchssteiner, "Integrable theory of the perturbation equations," Chaos, Solitons and Fractals, vol. 7, no. 8, pp. 1227-1250, 1996.

[8] Y. F. Zhang and H. Q. Zhang, "A direct method for integrable couplings of TD hierarchy," Journal of Mathematical Physics, vol. 43, no. 1, pp. 466-472, 2002.

[9] W. X. Ma, "Integrable couplings of vector AKNS soliton equations," Journal of Mathematical Physics, vol. 46, no. 3, Article ID 033507, 19 pages, 2005.

[10] W. X. Ma, "Enlarging spectral problems to construct integrable couplings of soliton equations," Physics Letters A, vol. 316, no. 1-2, pp. 72-76, 2003.

[11] F. Guo, Y. Zhang, and Q. Yan, "New simple method for obtaining integrable hierarchies of soliton equations with multicomponent potential functions," International Journal of Theoretical Physics, vol. 43, no. 4, pp. 1139-1146, 2004.

[12] Y. F. Zhang and X. X. Xu, "A trick loop algebra and a corresponding Liouville integrable hierarchy of evolution equations," Chaos, Solitons and Fractals, vol. 21, no. 2, pp. 445-456, 2004.

[13] H. Dong, X. R. Wang, and W. Zhao, "A new 4-dimensional implicit vector-form loop algebra with arbitrary constants and the corresponding computing formula of constant $\gamma$ in the variation identity," Applied Mathematics and Computation, vol. 218, no. 22, pp. 10998-11008, 2012.

[14] W. X. Ma, X. X. Xu, and Y. F. Zhang, "Semi-direct sums of Lie algebras and continuous integrable couplings," Physics Letters A, vol. 351, no. 3, pp. 125-130, 2006.

[15] H. X. Yang and X. X. Xu, "Integrable expanding models for discrete systems application: coupled TODa and relativistic TODa lattice," International Journal of Modern Physics B, vol. 19, no. 13, pp. 2121-2128, 2005.

[16] Y. Q. Yao, J. Ji, D. Y. Chen, and Y. B. Zeng, "The quadraticform identity for constructing the Hamiltonian structures of the discrete integrable systems," Computers \& Mathematics with Applications, vol. 56, no. 11, pp. 2874-2882, 2008.

[17] W.-X. Ma, "A discrete variational identity on semi-direct sums of Lie algebras," Journal of Physics A: Mathematical and Theoretical, vol. 40, no. 50, pp. 15055-15069, 2007.

[18] V. K. Mel'Nikov, “On equations for wave interactions," Letters in Mathematical Physics, vol. 7, no. 2, pp. 129-136, 1983.

[19] F. Yu and L. Li, "A blaszak-marciniak lattice hierarchy with selfconsistent sources," International Journal of Modern Physics B, vol. 25, no. 25, pp. 3371-3379, 2011. 
[20] F. J. Yu, "Non-isospectral integrable couplings of AblowitzLadik hierarchy with self-consistent sources," Physics Letters A, vol. 372, no. 46, pp. 6909-6915, 2008.

[21] T.-C. Xia, "Two new integrable couplings of the soliton hierarchies with self-consistent sources," Chinese Physics B, vol. 19, no. 10, Article ID 100303, 2010.

[22] H.-W. Yang, H.-H. Dong, and B.-S. Yin, "Nonlinear integrable couplings of a nonlinear Schrödinger - Modified Korteweg de Vries hierarchy with self-consistent sources," Chinese Physics B, vol. 21, no. 10, Article ID 100204, 2012.

[23] H. W. Yang, H. H. Dong, B. S. Yin, and Z. Y. Liu, "Nonlinear bi-integrable couplings of multicomponent Guo hierarchy with self-consistent sources," Advances in Mathematical Physics, vol. 2012, Article ID 272904, 14 pages, 2012.

[24] Y. B. Zeng, W. X. Ma, and R. L. Lin, "Integration of the soliton hierarchy with self-consistent sources," Journal of Mathematical Physics, vol. 41, no. 8, pp. 5453-5489, 2000.

[25] Y. B. Zeng, "New factorization of the Kaup-Newell hierarchy," Physica D, vol. 73, no. 3, pp. 171-188, 1994.

[26] Y. Huang, Y. Zeng, and O. Ragnisco, "The Degasperis-Procesi equation with self-consistent sources," Journal of Physics A: Mathematical and Theoretical, vol. 41, no. 35, Article ID 355203, 2008. 


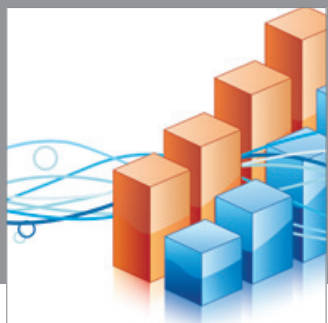

Advances in

Operations Research

mansans

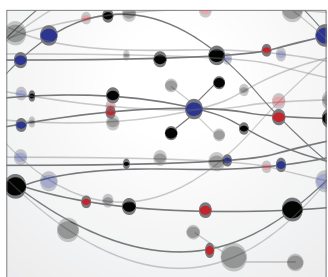

The Scientific World Journal
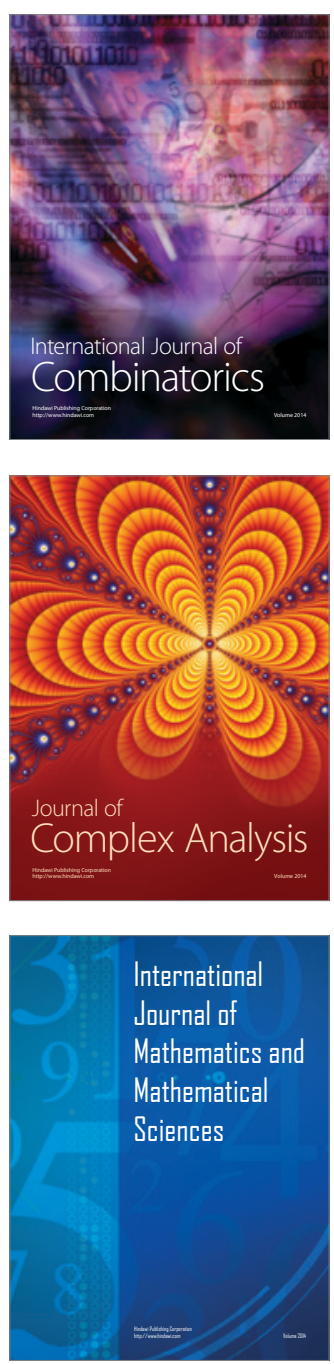
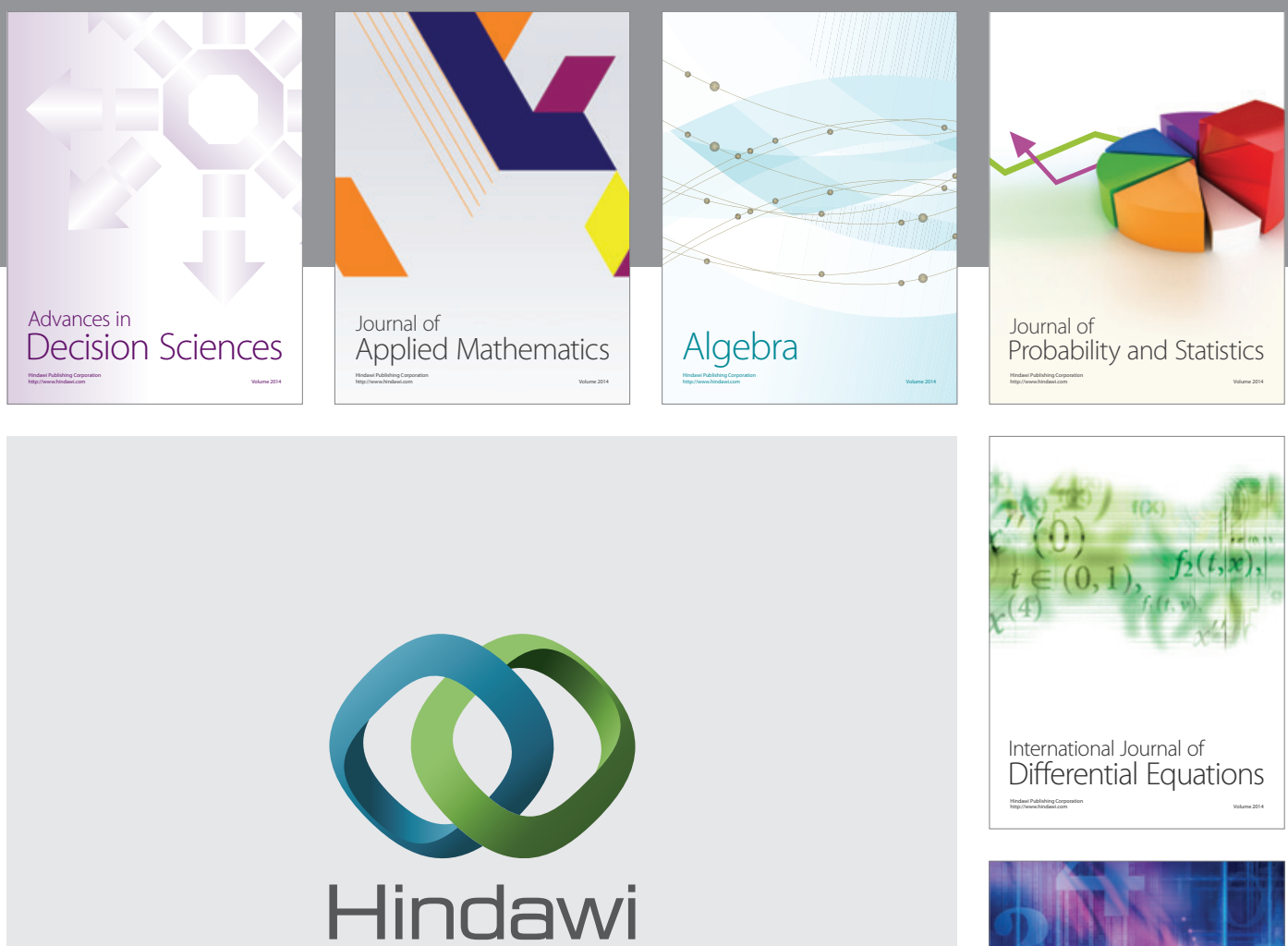

Submit your manuscripts at http://www.hindawi.com
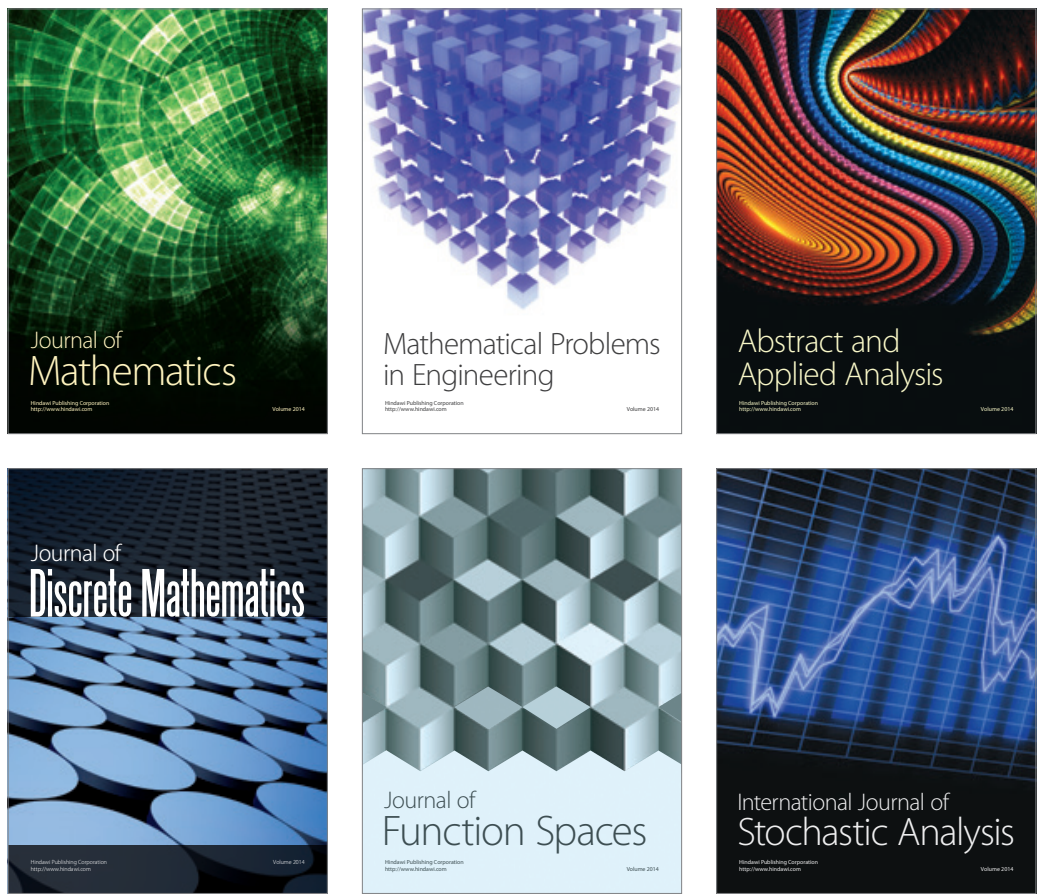

Journal of

Function Spaces

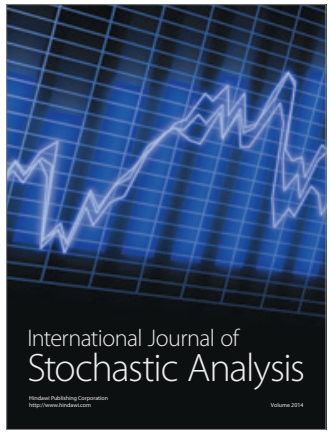

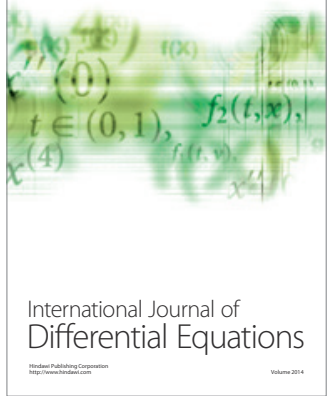
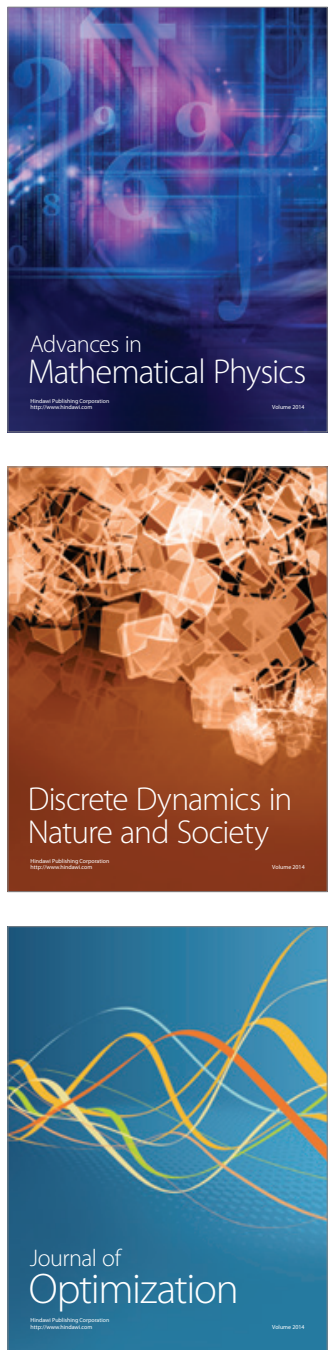